\title{
Exercise and Peripheral Nerve Grafts as a Strategy To Promote Regeneration after Acute or Chronic Spinal Cord Injury
}

\author{
Catherine C. Theisen,' Rahul Sachdeva, Scarlett Austin,' Danielle Kulich, \\ Victoria Kranz, and John D. Houle ${ }^{1}$
}

\begin{abstract}
Therapeutic interventions after spinal cord injury (SCI) routinely are designed to address multiple aspects of the primary and/ or secondary damage that occurs. Exercise has a demonstrated efficacy for post-SCI complications such as cardiovascular dysfunction, neuropathic pain, and chronic inflammation, yet there is little understanding of the mechanisms by which improvements might result from this non-invasive approach. Here we review several of our observations of molecular and cellular changes within the injured spinal cord following acute or delayed exercise regimens that illustrate the potential for positive effects on neuroprotection and rehabilitation. Further, we provide new information about the role of exercise in promoting the regeneration of spinal axons into peripheral nerve grafts (PNGs) placed immediately or 6 weeks after injury. Acute and chronically injured propriospinal neurons within the lumbar spinal cord displayed the greatest propensity for enhanced regeneration after exercise, which correlates with the direct sensory input to this region from exercised hindlimb muscles. Future studies will extend these observations by testing whether exercise will boost the regenerative effort of axons to extend beyond the graft, interact with intraspinal targets, and establish functional connections across a lesion.
\end{abstract}

Keywords: exercise; regeneration; transplantation

$\mathbf{R}$ ECOVERY OF FUNCTION is limited following spinal cord injury (SCI), due to primary trauma and necrosis, and secondary damage resulting in neurotoxicity, vascular dysfunction, inflammation, formation of the glial scar, demyelination, neurotoxicity, and apoptosis. The eminent opinion in the field is that neuroprotection, regeneration, and rehabilitation will require a combination treatment strategy, potentially geared to different stages and processes of the injury response. Here we highlight two strategies, exercise and peripheral nerve grafts (PNGs), and the intersection of their mechanisms and evidence of combinatorial benefits. Whereas exercise appears to be beneficial through a variety of cellular and biochemical mechanisms, PNGs provide a hospitable environment to attract and support growth of regenerating axons, and there is emerging evidence that the combination of the two strategies may work additively. ${ }^{1-3}$

\section{Exercise-dependent neuroplasticity}

A number of studies over the past several decades have shown the positive neurorehabilitative and neuroplasticity-associated effects of exercise and training after SCI. Following SCI, loss of integration of descending pathway control on afferent stimulation and input to the cord can lead to a number of maladaptive developments, including loss of habituation in motor neurons, which can result in hyperreflexia. Exercise has been shown to at least partially ameliorate these effects. In a thoracic transection rat model, cycling exercise restored frequency-dependent depression of the H-reflex, prevented hindlimb muscle atrophy, and led to maintenance of motoneuron resting membrane potential. ${ }^{4-6}$ Exercise beginning within the first week after SCI in a cervical contusion rat model effectively prevents maladaptive plasticity of nociceptive primary afferents and associated tactile allodynia. ${ }^{7}$ Recently, a peripheral nerve injury model linked this reduction of neuropathic pain and prevention of nociceptor sprouting seen with early exercise to normalization of microglial neurotrophic factor expression and chloride cotransporter homeostasis in the dorsal horn. ${ }^{8}$ Exercise and locomotor training has been shown to improve recovery after SCI in a variety of animal injury models and has been a staple of rehabilitation techniques in the clinic. ${ }^{9-11}$

Much of the existing literature exploring the impact of exercise following injury focuses on the role of neurotrophic factors on neuroprotection and activity-dependent neuroplasticity. Neurotrophic

\footnotetext{
${ }^{1}$ Department of Neurobiology and Anatomy, Drexel Spinal Cord Research Center, Drexel University College of Medicine, Philadelphia, Pennsylvania.

${ }^{2}$ University of British Columbia, Autonomic Research Laboratory, International Collaboration on Repair Discoveries (ICORD), Vancouver, British Columbia, Canada.
} 
factors have long been known to provide neuroprotective support to neurons following central or peripheral insult to axons or cell bodies. BDNF, NT-3, and NT-4 all promote survival and growth of spinal neurons after injury ${ }^{12,13}$ and when applied specifically in rodent models of SCI, these neurotrophic factors are capable of preventing neuronal cell death after axotomy. ${ }^{14}$ Studies in uninjured rats have shown that acute and prolonged voluntary exercise increases BDNF and NT-3 in cord motoneurons and skeletal muscle. ${ }^{15}$ Subsequently, through paralysis of hindlimb muscles with botulinum toxin, it was shown that neuromuscular activity is required for increased BDNF and TrkB expression with exercise. ${ }^{16}$ Additional studies demonstrated that increased BDNF expression with exercise leads to induction of intraspinal CREB, synapsin I, and GAP-43 messenger ribonucleic acids (mRNAs) and potential modulation of synaptic plasticity. ${ }^{17,18}$ In the context of SCI, our group and others have shown similar positive effects of exercise on neurotrophic factor expression. In a rat low thoracic transection model, we observed an exercisedependent increase in neurotrophic factors such as BDNF, NT-3, and NT-4, in the lumbar and thoracic cord below the lesion. This finding has been demonstrated, using several models of exercise or training, including treadmill training, wheel training, and cycling exercise and is correlated with a beneficial modulation of spinal reflexes as measured by return of frequency-dependent depression during H-reflex testing. ${ }^{19}$ We also observed increased levels of GDNF and NT3 in the thoracic cord above the lesion and Graziani and colleagues ${ }^{20}$ detected increased BDNF levels in the somatosensory cortex of transectedexercised rats and cortical reorganization in response to cycling, indicating a more broad effect of exercise, beyond spinal cord regions receiving direct sensory input from exercised hindlimbs. ${ }^{21}$

Table 1 indicates similarities and differences of some of the modalities and schedules of our diverse exercise paradigms after SCI that may be useful in predicting recovery. Certainly, the level and severity of injury play significant roles in choosing a particular exercise or training regimen, as spinal transected rats require considerable assistance during cycling and treadmill walking, whereas less severely injured (cervical unilateral contusion) rats will eventually use all four limbs during an exercise such as forced wheel walking. We routinely initiate our exercise regimens at 5 days after SCI after observing that exercise at shorter post-injury intervals (1-3 days) leads to exaggerated muscle fatigue, muscle degeneration, and generally diminished functional outcome (unpublished observations). Interestingly, the length of each bout of a particular type of exercise can be quite variable (5-60 min) without negatively effecting the pattern or level of recovery. It is interesting to note that some outcome measures (e.g., increased neurotrophic factor production) are constant, regardless of the type or extent of exercise or whether the onset of exercise was acute or delayed.
In our rat transection injury model, exercise after injury results in decreased expression of apoptosis-associated microRNAs (miRNAs) and decreased caspase protein expression in the injured cord, suggestive of a neuroprotective effect. ${ }^{22}$ This correlated with increased cFos (a marker of synaptic activity) expression in the dorsal horn and intermediate gray, indicating a potential for neuroplasticity in the spinal cord after exercise. ${ }^{23}$ There is evidence that these effects of exercise vary between acute bouts and prolonged periods of exercise, as BDNF expression in the injured cord is increased with 5 days of exercise above what occurs with 1 day of exercise (unpublished data). In the lumbar spinal cord acute and prolonged exercise increased expression of mRNA for BDNF and GDNF, increased heat-shock protein expression, and decreased caspase protein expression. ${ }^{24}$ In motoneurons and intermediate gray neurons specifically, acute and prolonged exercise increased levels of mRNA for BDNF, GDNF, and NT-4. However, in large DRG neurons, mRNA for neurotrophic factors was unchanged and only caspase mRNA expression was decreased by exercise. This suggests that specific neuron types may be more sensitive or responsive to exercise.

There are molecular changes associated with exercise, which led us to believe exercise could be useful in facilitating regeneration. Recent studies highlighted the potential for boosting the intrinsic regenerative potential of injured central nervous system (CNS) neurons. Deletion of negative regulators of mammalian target of rapamycin (mTOR), such as phosphatase and tensin homolog (PTEN) or tuberous sclerosis protein 1 (TSC1), facilitates remarkable improvement in regeneration of optic and corticospinal tract axons. ${ }^{22,25,26} \mathrm{We}$ have shown that cycling exercise increases mTOR and decreases PTEN mRNA and protein levels in the injured spinal cord and increases activation of the ribosomal protein S6 in intermediate gray (presumptively propriospinal) neurons. ${ }^{27}$ Additionally, the neurotrophic factors increased by exercise likely contribute to axonal regeneration after injury. In rodent models, BDNF delivered to the spinal cord after injury can promote regenerative growth of injured axons, and transplants treated with neurotrophic factors increase regeneration and locomotor recovery of function following SCI. ${ }^{28,29}$ Recently, we demonstrated that BDNF treatment applied to PNGs enhances the integration of chronically injured axons that regenerated through the grafts back into a chondroitinase-treated SCI site. ${ }^{30}$

\section{Cautionary tales of exercise-related aberrant recovery}

Although the majority of studies support the concept that exercise is beneficial after SCI, there are indications that some negative effects may result, especially when testing for recovery of a specific

Table 1. Variability in Exercise Training after SCI

\begin{tabular}{llll}
\hline Parameter & Forced wheel & Cycling & Treadmill \\
\hline Level and type of Injury & $\mathrm{C} 5 \mathrm{Ct}$ & $\mathrm{T} 12 \mathrm{Tx}$ & $\mathrm{T} 12 \mathrm{Tx}$ \\
Initial bout & $5 \mathrm{dpi}$ & $5 \mathrm{dpi}$ & $5 \mathrm{dpi}$ \\
Frequency & $20 \mathrm{~min}$ daily & $15-60 \mathrm{~min}$ daily & $5-15 \mathrm{~min}$ daily \\
Efficacy when delayed & Yes & Yes & Yes \\
Body weight supported & No & Yes & Yes \\
Muscle loading & Yes & No & Yes \\
Passive or active movement & Active & Passive & Active \\
Intensity & $6.0-14.0 \mathrm{~m} / \mathrm{min}$ & $45 \mathrm{rpm}$ & $1.0-6.0 \mathrm{~m} / \mathrm{min}$ \\
\hline
\end{tabular}

C, cervical; Ct, contusion; dpi, days post-injury; m/min, meters per minute; rpm, rotations per minute; SCI, spinal cord injury; T, thoracic; Tx, transection. 
task when the exercise/training was designed to elicit a specific result. In a ground-breaking study by Edgerton and associates ${ }^{31}$ spinalized cats trained to walk on a treadmill were not able to stand, and when these cats were then trained to stand their stepping declined. This suggested that spinal cord learning might be restricted to a single task at a time. From the laboratory of Karim Fouad, rats performed worse on the horizontal ladder when they were trained only for a pellet-reaching task $\mathrm{k}^{32}$ and there was a negative impact on motor performance of the contralateral forelimb when the SCIaffected forelimb received both specific and general training. ${ }^{33}$ The authors proposed the possibility of competition for spared circuitry between the forelimbs and the tasks being trained for. In a study designed to take advantage of a combination of exercise and chondroitinase-induced plasticity, Garcia-Alias and colleagues ${ }^{34}$ demonstrated enhanced manual dexterity when rats received taskspecific training, but this reaching skill was diminished in rats that received only general locomotor training (ladder walking). This presents a conundrum for the future design of therapeutic strategies when we seem to be unsure of the most effective use of exercise/ training in conjunction with a pharmacological treatment.

\section{Exercise-dependent regeneration-acute grafting}

We recently applied our method of cycling exercise following low thoracic (T12) transection $^{35}$ to test whether exercise would indeed promote axon regeneration. For this study adult rats received an acute PNG to support regrowth of ascending spinal axons and began cycling exercise 5 days later. Five weeks post-injury the retrograde tracer True Blue (TB) was applied to the distal end of the PNG, as a means to identify and count the number of neuronal cell bodies caudal to the injury that regenerated an axon into the ascending PNG. We found that 4 weeks of cycling improved regeneration into a PNG from thoracic and lumbar intermediate gray neurons. The majority of regenerating neurons in exercised and non-exercised groups were within $5 \mathrm{~mm}$ of the lesion and exercise increased their numbers by $70 \%$. The area where exercise showed the most dramatic effect was in more distant lumbar cord, where the number of regenerating neurons increased by $700 \%$. Interestingly, although lumbar-level dorsal root ganglion neurons (DRGs) did regenerate axons into the PNG, there was no exercise-associated increase in regeneration by these primary sensory neurons. We further characterized this cell-type specific effect of exercise using fluorescent in situ hybridization and confocal microscopy to detect an increase in levels of mRNAs encoding the regeneration associated genes (RAGs), such as GAP43, $\beta$-actin and neuritin. Propriospinal neurons showed increased mRNA expression of RAGs in response to grafting and exercise, whereas DRGs increased RAG expression only in the presence of a graft. This work established a molecular basis for exercise-dependent regeneration and an interesting dichotomy in the response of DRGs to axotomy and exercise. Our results indicate no improvement in the regenerative effort after injury to the centrally projecting process of DRGs with exercise, even though there is solid evidence of the positive effects of exercise on axon regeneration by cultured sensory neurons or after peripheral nerve crush. ${ }^{36}$

Having established that exercise is associated with an increase in regeneration by neurons that may be most directly affected by sensory feedback from rhythmic limb movement during cycling, we asked whether exercise might also effect the regenerative effort of neurons more distant from the area of direct stimulation. We utilized the same techniques as previously described: an acute PNG, 4 weeks of cycling exercise beginning 5 days after a low thoracic transection in rats, and retrograde tracing to identify neurons regenerating their axon into a peripheral nerve graft $^{35}$; however, we apposed the PNG to the rostral lesion cavity, in order to study regeneration by neurons located rostral to the lesion site (Fig. 1). In the T11 spinal cord segment (the 5-mm segment immediately rostral to the descending PNG), we found that exercise significantly increased the number of regenerating neurons extending axons into the graft from the T11 segment (2809.0 \pm 957.5 vs. $1190.1 \pm 782.6$, Fig. 2), which was a $230 \%$ increase. As was the case in the earlier study, most regenerating neurons were interneurons and there was a gradual decline in the number of neurons as the distance from the graft-spinal cord interface increased. In the more distant, upper thoracic cord (T1-T10 segments), labeled neurons were distributed throughout the rostral-caudal extent of this block with many in close proximity to the central canal (Fig. 2). In the upper thoracic cord, exercise increased the number of neurons regenerating axons into the PNG compared with their nonexercised counterparts $(508.8 \pm 97.06$ vs. $164.1 \pm 104.4)$, a $300 \%$ increase. This observation of exercise increasing regeneration in neurons as far as the upper thoracic cord seems to align with findings from previous studies that demonstrated increases in molecules related to neuroprotection and neuroregeneration at spinal cord locations outside of the area directly stimulated by exercise or across lesion sites. More studies are needed to determine which particular molecules and pathways are specifically expressed locally or across the cord and whether certain mediators affect certain types of regeneration or recovery.

\section{Exercise-dependent regeneration-delayed grafting}

Despite extensive knowledge of the effects of exercise in the acute SCI situation, relatively little is known about possible beneficial effects of exercise in a chronic injury condition. Increases in BDNF levels in the lumbar cord are observed with both acute and delayed hindlimb exercise, ${ }^{23}$ but whether this would translate to any anatomical or functional evidence of increased axonal regeneration in the chronically injured spinal cord remained to be investigated.

To address this issue we performed a study in which adult rats received a T12 transection injury, delayed PNGs for support of ascending (a) and descending (d) axons and acute or delayed exercise (Fig. 1). At 6 weeks after the initial SCI the transection site was re-exposed, scar tissue was removed with gentle aspiration, and aPNGs and dPNGs were apposed to the injured spinal cord below and above the lesion to facilitate axon growth across the caudal and rostral graft-host interfaces, respectively. We aimed to test whether the effects of exercise are temporally variable (i.e., whether therapeutically relevant mechanisms of exercise interact with the dynamic environment of the spinal cord or changes in the intrinsic ability of neurons to regenerate from the acute to chronic post-injury period). Because the clinical barriers to exercise in the acute and subacute period post-SCI are significant and therapeutic relevance of exercise would be limited if its efficacy was limited to an acute post-injury period, it was important to determine whether there were significant differences on regeneration of acute onset exercise compared with delayed onset of exercise. Therefore, some animals in this study began exercise acutely after transection injury (5 days post-SCI), whereas others began exercise at a chronic period ( 5 weeks post-SCI, 1 week prior to nerve grafting). TB was applied at the distal end of the grafts 5 weeks after grafting.

In quantifying the neurons caudal to the lesion site that regenerated their axons into the aPNG, we again parceled neurons in 
A

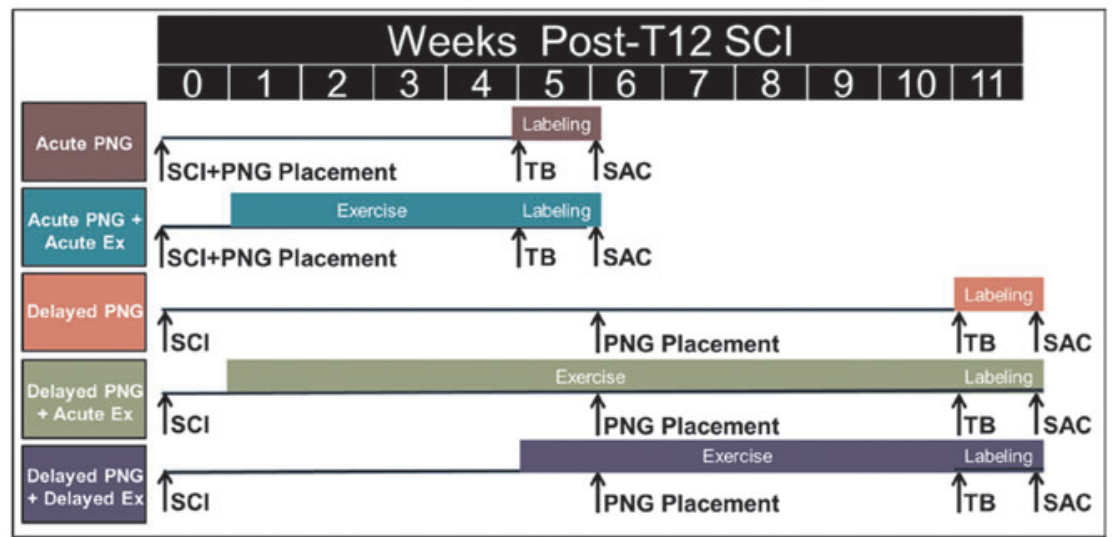

B
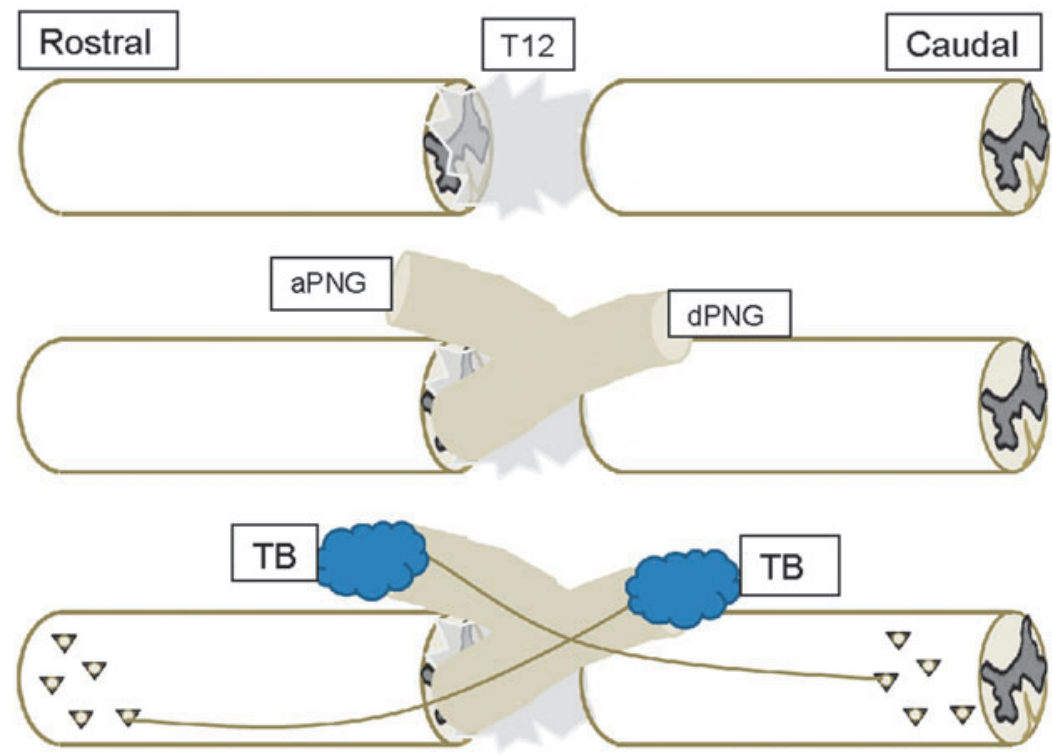

FIG. 1. Time line and schematic of injury, grafting, and exercise. (A) Time line of experiments showing acute PNGs placed at the time of SCI and chronic PNGs placed 6 weeks post-SCI. Exercise begins either acutely 5 days after initial SCI or with a delay at 5 weeks post-SCI, and it continues until the time of sacrifice. Animals are euthanized at 6 weeks post-SCI in acute PNG experiments or 12 weeks post-SCI in chronic PNG experiments. TB retrograde tracer is applied to distal ends of PNGs 5 days before euthanasia in all experiments. The group colors correspond to the colored bars in Figure 2. (B) Schematic showing initial SCI, apposition of aPNG to caudal lesion boundary and dPNG to rostral lesion boundary, and TB labeling of distal ends of PNGs in order to retrogradely label cell bodies of neurons extending axons into the grafts. aPNG, ascending PNG; dPNG, descending PNG; PNG, peripheral nerve graft; SCI, T12 spinal cord transection injury; TB, True Blue retrograde tracer.

two regions: the 5-mm segment (T13 cord) immediately caudal to the lesion and the more distal lumbar cord (L1-L5). Neither acute exercise nor delayed exercise led to an increase in regenerating neurons in the T13 segment compared with non-exercised controls (Fig. 2). In the lumbar cord a significant increase was seen in the number of neurons regenerating axons into the aPNG in both the acute exercise chronic PNG recipient group and the delayed exercise chronic PNG recipient group when compared with the non-exercised chronic PNG control group (1346 \pm 303.1 and $1660 \pm 699.5$ vs. $690.7 \pm 365.7)$, indicating that there was no benefit to having a longer pre-graft period of exercise. In the chronic dPNG, neither the 5-mm segment (T11 cord) immediately rostral to the lesion and dPNG nor the more rostral T1-T10 upper thoracic regions showed any significant increase in regenerating neurons between acute exercise, delayed exercise, and no exercise groups.
The new data presented here show that hindlimb cycling exercise has a positive effect on increasing regeneration into PNGs from chronically injured neurons, specifically by propriospinal neurons in the lumbar cord. No significant differences in regeneration into delayed PNGs were seen between rats that were exercised beginning acutely post-SCI and rats beginning exercise in a chronically SCI period. This suggests that there is no long lasting or cumulative effect of exercise when axon regeneration is used as the benchmark for success. This result demonstrates that exercise has the potential to facilitate regeneration in a therapeutically relevant time course, without the clinically limiting necessity to begin in the acute or subacute period after SCI.

Interestingly, these results were markedly different from the effect of exercise on regeneration into an acute graft. In the acute PNG experiments, exercise significantly increased acute regeneration in 

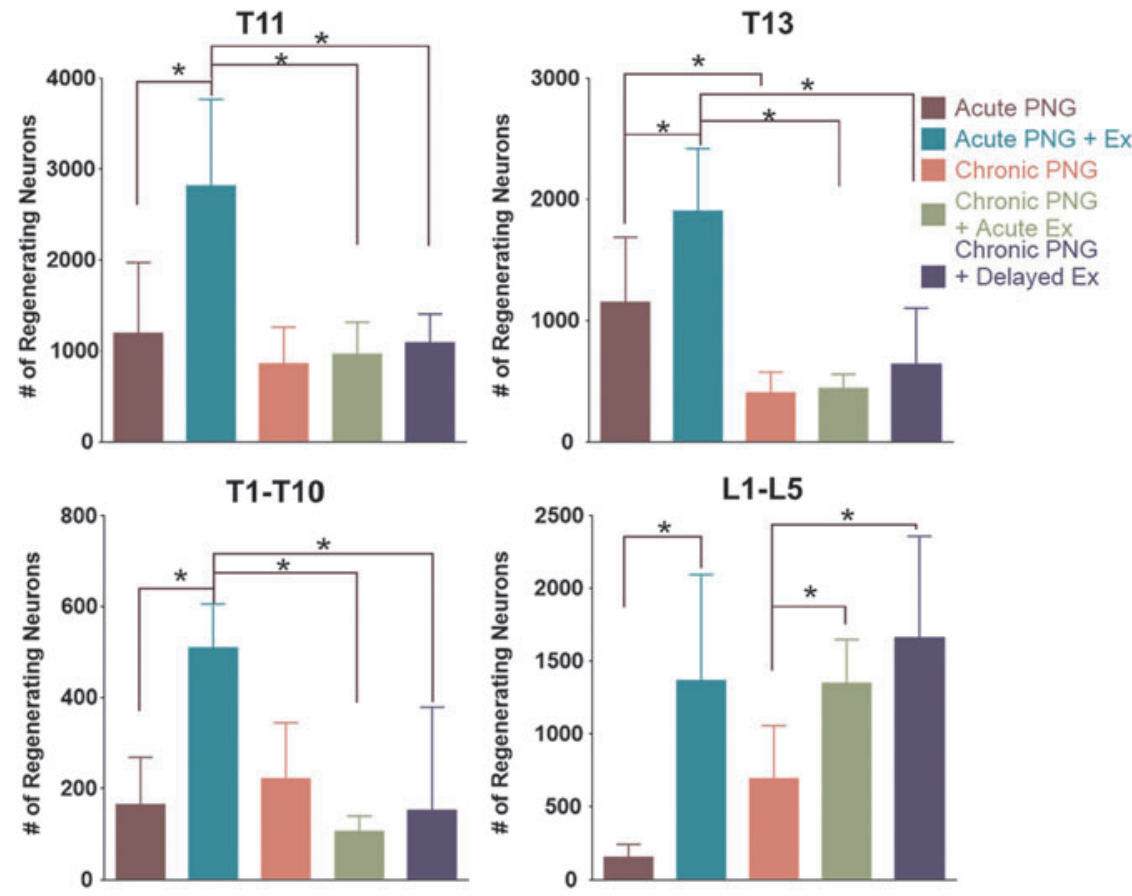

FIG. 2. Quantification of TB positive neurons in spinal cord segments rostral and caudal to the lesion. Top left: T11 thoracic spinal cord neurons labeled by axons extending into the dPNG. Top right: T13 thoracic spinal cord neurons labeled by axons extending into the aPNG. Bottom left: T1-T10 thoracic spinal cord neurons labeled by axons extending into the dPNG. Bottom right: L1-L5 lumbar spinal cord neurons labeled by axons extending into the aPNG (error bars reflect standard deviation [SD]; *indicates $p<0.05$ ).

all thoracic and lumbar spinal cord regions. Acute regeneration was increased by $70 \%$ in the T11 and T13 cord segments above and below the lesion, by $300 \%$ in the T1-T10 upper thoracic cord, and by $900 \%$ in L1-L5 lumbar cord. By comparison, in the delayed PNG experiment, both acute and delayed exercise increased regeneration by chronically injured neurons by $\sim 200 \%$, but only in a single region, the L1-L5 lumbar cord. At this point we can only speculate that direct sensory input from exercised hindlimbs to the lumbar spinal cord has a prominent role in enhancing the regenerative effort of chronically injured neurons in this region.

Exercise appears to affect the intrinsic potential of neurons to regenerate after injury through upregulation of mediators of growth, such asthe PTEN/mTOR pathway and RAGs such as GAP43, $\beta$-actin and neuritin. ${ }^{27,35}$ This exercise-dependent increase in RAGs in spinal interneurons was associated with an increase in regeneration by acutely injured neurons and it is possible chronically injured neurons do not respond in a similar manner or to the level necessary to promote axonal regrowth. Our finding that exercise seems to have a much more robust impact on regeneration in acutely injured neurons would suggest a critical time period after injury for the beneficial effects of exercise.

The extrinsic environment of the spinal cord changes dramatically with SCI and secondary degenerative changes such as glial scarring after injury. We and others have shown that local BDNF and NT-3 levels are downregulated after spinal cord contusion or transection, but these same neurotrophic factors can be increased in the injured spinal cord and the peripheral muscle with treadmill training, cycle training, motorized running wheels. or with voluntary locomotion in freely moving running wheels. PNGs provide structural support to bypass inhibitory features of an acute or chronic injury site and physiological support in the form of neurotrophic factors to support the intrinsic growth capacity of injured neurons. Exercise likely augments these basic levels of support by affecting both intrinsic and extrinsic regulators of axonal growth.

Future studies are needed to investigate both the molecular mechanisms by which exercise may facilitate regeneration and its potential to support reintegration of regenerating axons beyond a distal graft-host interface. Currently, we are examining segmental differences in neurotrophic factor production and markers of synaptic activity seen with exercise in the chronically injured cord, as it relates to the acutely injured cord. Having established a positive influence of exercise on axon regeneration into acute and delayed PNGs we are examining the possible influence of exercise on outgrowth and functional reintegration of these axons with the host cord. Fewer than $10 \%$ of axons that enter these grafts typically extend beyond the distal end and even with modulation of extracellular matrix with chondroitinase treatment this increases to less than $20 \%$ of the total within the graft, therefore growth beyond the graft of about $80 \%$ of axons is being thwarted. Knowledge of many of the beneficial effects of exercise (such as increasing local neurotrophic factor levels, modulating the PTEN/mTOR pathway, and increasing synaptic activity of possible target neurons) makes it reasonable to explore the potential role for exercise to drive the regenerative effort of more axons beyond the graft-host interface and to facilitate interaction of regenerating axons with intraspinal targets.

\section{Acknowledgments}

This work was supported by NIH NS055976 and the Craig H. Neilsen Foundation (224125).

\section{Author Disclosure Statement}

No conflicting financial interests exist. 


\section{References}

1. Richardson, P.M., McGuinness, and U.M., Aguayo, A.J. (1980). Axons from CNS neurons regenerate into PNS grafts. Nature 284, 264 265.

2. David, S., and Aguayo, A.J. (1981). Axonal elongation into peripheral nervous system "bridges" after central nervous system injury in adult rats. Science 214, 931-933.

3. Tom, V.J., Sandrow-Feinberg, H.R., Miller, K., Santi, L., Connors, T. Lemay, M.A., and Houle, J.D. (2009). Combining peripheral nerve grafts and chondroitinase promotes functional axonal regeneration in the chronically injured spinal cord. J. Neurosci. 29, 14881-14890.

4. Skinner, R.D., Houle, J.D., Reese, N.B., Berry, C.L., and Garcia-Rill, E. (1996). Effects of exercise and fetal spinal cord implants on the Hreflex in chronically spinalized adult rats. Brain Res. 729, 127-131.

5. Peterson, C.A., Murphy, R.J., Dupont-Versteegden, E.E., and Houle, J.D. (2000). Cycling exercise and fetal spinal cord transplantation act synergistically on atrophied muscle following chronic spinal cord injury in rats. Neurorehabil. Neural Repair 14, 85-91.

6. Beaumont, E., Houle, J.D., Peterson, C.A., and Gardiner, P.F. (2004). Passive exercise and fetal spinal cord transplant both help to restore motoneuronal properties after spinal cord transection in rats. Muscle Nerve 29, 234-242.

7. Detloff, M.R., Smith, E.J., Quiros Molina, D., Ganzer, P.D., and Houle, J.D. (2014.) Acute exercise prevents the development of neuropathic pain and the sprouting of non-peptidergic (GDNF- and artemin-responsive) c-fibers after spinal cord injury. Exp. Neurol. 255, $38-48$.

8. Lopez-Alvarez, V.M., Modol, L., Navarro, X., and Cobianchi, S. (2015). Early increasing-intensity treadmill exercise reduces neuropathic pain by preventing nociceptor collateral sprouting and disruption of chloride cotransporters homeostasis after peripheral nerve injury. Pain 156,1812-1825.

9. Jakeman, L.B., Hoschouer, E.L., and Basso, D.M. (2011). Injured mice at the gym: review, results and considerations for combining chondroitinase and locomotor exercise to enhance recovery after spinal cord injury. Brain Res. Bull. 84, 317-326.

10. Harkema, S.J., Hillyer, J., Schmidt-Read, M., Ardolino, E., Sisto, S.A., and Behrman, A.L. (2012). Locomotor training: as a treatment of spinal cord injury and in the progression of neurologic rehabilitation. Arch. Phys. Med. Rehabil. 93, 1588-1597.

11. Cohen-Adad, J., Martinez, M., Delivet-Mongrain, H., and Rossignol, S. (2014). Recovery of locomotion after partial spinal cord lesions in cats: assessment using behavioral, electrophysiological and imaging techniques. Acta Neurobiol. Exp. (Wars) 74,142-157.

12. Koliatsos, V.E, Clatterbuck, R.E., Winslow, J.W., Cayouette, M.H., and Price, D.L. (1993). Evidence that brain-derived neurotrophic factor is a trophic factor for motor neurons in vivo. Neuron 10, 359 367 .

13. Friedman, B., Kleinfeld, D., Ip, N.Y., Verge, V.M., Moulton, R., Boland, P., Zlotchenko, E., Lindsay, R.M., and Liu, L. (1995). BDNF and NT-4/5 exert neurotrophic influences on injured adult spinal motor neurons. J. Neurosci. 15,1044-1056.

14. Giehl, K.M., and Tetzlaff, W. (1996). BDNF and NT-3, but not NGF, prevent axotomy-induced death of rat corticospinal neurons in vivo. Eur. J. Neurosci. 8, 1167-1175.

15. Gomez-Pinilla, F., Ying, Z., Opazo, P., Roy, R.R., and Edgerton, V.R. (2001). Differential regulation by exercise of BDNF and NT-3 in rat spinal cord and skeletal muscle. Eur. J. Neurosci. 13,1078-1084.

16. Gomez-Pinilla, F., Ying, Z., Roy, R.R., Molteni, R., and Edgerton, V.R. (2002). Voluntary exercise induces a BDNF-mediated mechanism that promotes neuroplasticity. J. Neurophys. 88, 2187-2195.

17. Gomez-Pinilla, F., Ying, Z., Roy, R.R., Hodgson, J., and Edgerton, V.R. (2004). Afferent input modulates neurotrophins and synaptic plasticity in the spinal cord. J. Neurophys. 92, 3423-3432.

18. Ying, Z., Roy, R.R., Edgerton, V.R., and Gomez-Pinilla, F. (2005). Exercise restores levels of neurotrophins and synaptic plasticity following spinal cord injury. Exp. Neurol. 193, 411-419.

19. Cote, M.P., Azzam, G.A., Lemay, M.A., Zhukareva, V., and Houle, J.D. (2011). Activity-dependent increase in neurotrophic factors is associated with an enhanced modulation of spinal reflexes after spinal cord injury. J. Neurotrauma 28, 299-309.
20. Graziani, A., Foffani, G., Knuden, E.B., Shumsky, J., and Moxon, K.A. (2013). Passive exercise of the hind limbs after complete thoracic transection of the spinal cord promotes cortical reorganization. PLoS One 8, e54350.

21. Cote, M.P., Amin, A.A., Tom, V.J., and Houle, J.D. (2011). Peripheral nerve grafts support regeneration after spinal cord injury. Neurotherapeutics 8, 294-303.

22. Liu, G., Keeler, B.E., Zhukareva, V., and Houle, J.D. (2010). Cycling exercise affects the expression of apoptosis-associated microRNAs after spinal cord injury in rats. Exp. Neurol. 226, 200-206.

23. Houle, J.D., and Cote, M.P. (2013), Axon regeneration and exercisedependent plasticity after spinal cord injury. Ann. N. Y. Acad. Sci. 1279, 154-163.

24. Keeler, B.E., Liu, G., Siegfried, R.N., Zhukareva, V., Murray, M., and Houle, J.D. (2012). Acute and prolonged hindlimb exercise elicits different gene expression in motoneurons than sensory neurons after spinal cord injury. Brain Res. 1438, 8-21.

25. Park, K.K., Liu, K., Hu, Y., Smith, P.D., Wang, C., Cai, B., Xu, B., Connolly, L., Kramvis, I., Sahin, M., and He, Z. (2008). Promoting axon regeneration in the adult CNS by modulation of the PTEN/ mTOR pathway. Science 322, 963-966.

26. Park KK, Liu K, Hu Y, Kanter JL, and He Z (2010). PTEN/mTOR and axon regeneration. Exp. Neurol. 223, 45-50.

27. Liu, G., Detloff, M.R., Miller, K.N., Santi, L., and Houle, J.D. (2012). Exercise modulates microRNAs that affect the PTEN/mTOR pathway in rats after spinal cord injury. Exp. Neurol. 233, 447-456.

28. Coumans, J.V., Lin, T.T, Dai, H.N., MacArthur, L., McAtee, M., Nash, C., and Bregman, B.S. (2001). Axonal regeneration and functional recovery after complete spinal cord transection in rats by delayed treatment with transplants and neurotrophins. J. Neurosci. 21, 9334-9344.

29. Bregman, B.S., Coumans, J.V., Dai, H.N., Kuhn, P.L., Lynskey, J., McAtee, M., and Sandhu, F. (2002). Transplants and neurotrophic factors increase regeneration and recovery of function after spinal cord injury. Prog. Brain Res. 137, 257-273.

30. Tom, V.J., Sandrow-Feinberg, H.R., Miller, K., Domitrovich, C., Bouyer J., Zhukareva, V., Klaw, M.C., Lemay, M.A., and Houle, J.D. (2013). Exogenous BDNF enhances the integration of chronically injured axons that regenerate through a peripheral nerve grafted into a chondroitinase-treated spinal cord injury site. Exp. Neurol. 239, 91100.

31. Edgerton, V.R., de Leon, R.D., Tillakaratne, N., Recktenwald, M.R., Hodgson, J.A., and Roy, R.R. (1997). Use-dependent plasticity in spinal stepping and standing. Adv. Neurol. 72, 233-247.

32. Girgis, J., Merrett, D., Kirkland, S., Metz, G.A.S., Verge, V., and Fouad, K. (2007). Reaching training in rats with spinal cord injury promotes plasticity and task specific recovery. Brain 130, 2993-3003.

33. Weishaupt, N., Vavrek, R., and Fouad, K. (2013). Training following unilateral cervical spinal cord injury in rats affects the contralesional forelimb. Neurosci. Lett. 28, 77-81.

34. Garcia-Alias, G., Barkhuysen, S., Buckle, M., and Fawcett, J.W. (2009). Chondroitinase ABC treatment opens a window of opportunity for task-specific rehabilitation. Nature Neurosci. 12, 1145-1152.

35. Sachdeva, R., Theisen, C.C., Ninan, V., Twiss, J.L., and Houlé, J.D. (2016). Exercise dependent increase in axon regeneration into peripheral nerve grafts by propriospinal but not sensory neurons after spinal cord injury is associated with modulation of regenerationassociated genes. Exp. Neurol. 276, 72-82.

36. Molteni, R., Zheng, J.G., Ying, Z., Gomez-Pinilla, F., and Twiss, J.L. (2004). Voluntary exercise increases axonal regeneration from sensory neurons. Proc. Natl. Acad. Sci. U. S. A. 101, 8473-8478.

Address correspondence to: John D. Houle, PhD Department of Neurobiology and Anatomy Drexel Spinal Cord Research Center Drexel University College of Medicine Philadelphia, PA 19129

E-mail: jhoule@drexelmed.edu 